\title{
Scalp lesions in a 78-year-old woman
}
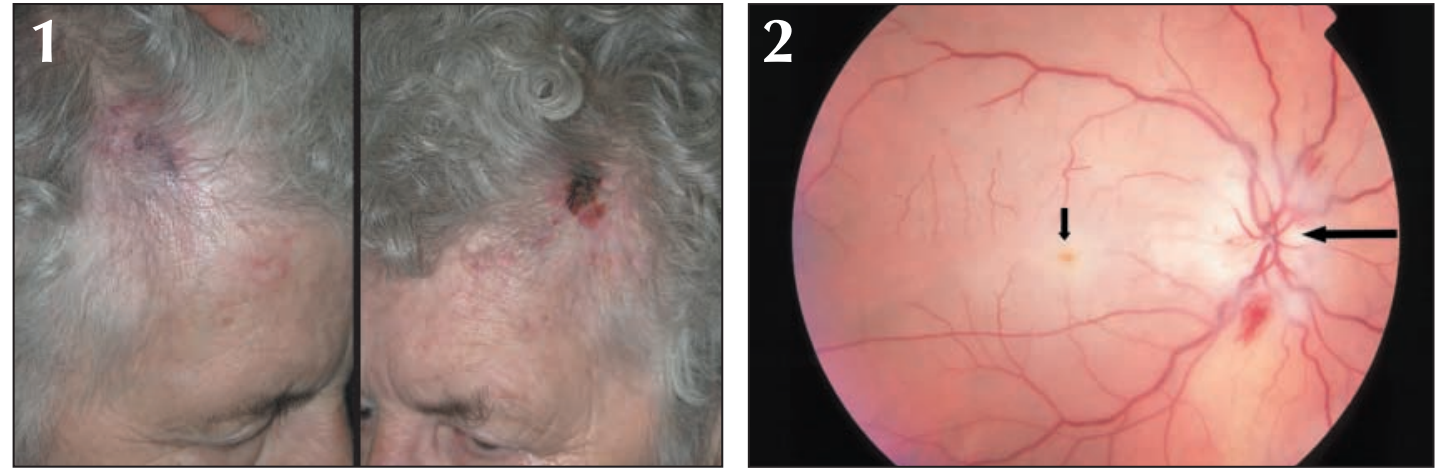

A 78-year-old woman presented to the emergency department with a 6-week history of temporal headaches, scalp lesions, jaw claudication and blurred vision in her right eye. Her erythrocyte sedimentation rate (ESR) was 20 (normal $\leq 20) \mathrm{mm} / \mathrm{h}$. She was presumed to have a herpes zoster infection and was discharged home with a prescription for oral valacyclovir therapy, fucidin cream and codeine. Her symptoms did not improve, and 1 week later she experienced sudden, painless loss of vision in her right eye.

During a neuro-ophthalmologic assessment, she described also experiencing scalp tenderness, neck pain, fatigue and anorexia in the weeks before her initial presentation. She had bilateral, erythematous, crusted scalp lesions with eschar formation (Fig. 1). Her temporal arteries were prominent and tender, without palpable pulses. She had no light perception in her right eye, and her visual acuity was 20/30 (6/9) in her left eye. Pupil testing revealed a relative afferent pupillary defect in the right eye. Funduscopy of the right eye demonstrated a pale, swollen optic nerve (Fig. 2, large arrow) as well as occlusion of the central retinal artery, as evidenced by $\infty$ retinal whitening with a central cherry red spot (Fig. 2, small arrow). Ophthalmic artery occlusion was diagnosed. The patient's ESR was only mildly elevated at $26 \mathrm{~mm} / \mathrm{h}$, but her C-reactive protein level was elevated at 29 (nor$\mathrm{mal} \leq 8) \mathrm{mg} / \mathrm{L}$. Biopsy of the right temporal artery was consistent with giant cell arteritis. High-dose intravenous steroid therapy led to resolution of her headaches and neck pain and healing of her scalp lesions over the next 4 weeks. Her vision in the left eye stabilized, but the right eye remained without light perception. One week after initiation of steroid therapy, her ESR was $1 \mathrm{~mm} / \mathrm{h}$, and her C-reactive protein level was less than $4 \mathrm{mg} / \mathrm{L}$. The steroid therapy was gradually tapered to an oral maintenance dose.

Giant cell arteritis, also known as temporal arteritis, is a systemic vasculitis affecting medium to large arteries, mainly the extracranial branches of the carotid artery. It occurs twice as often in women as in men, and the onset is typically after 55 years of age. ${ }^{1}$ Granulomatous inflammation in the vessel wall leads to obstruction of the lumen and ischemia in the territory distal to the occlusion. Presenting symptoms commonly include headache, scalp tenderness, jaw claudication, fatigue, decreased vision and myalgia (when associated with polymyalgia rheumatica). The most devastating complication is blindness in one or both eyes due to occlusion of the posterior ciliary arteries, which feed the optic nerve. Prompt recognition and treatment may prevent irreversible visual loss. Although the ESR is usually elevated, up to $10 \%$ of patients have a normal ESR. ${ }^{2}$ The C-reactive protein level is more sensitive than the ESR for detecting giant cell arteritis (100\% v. $92 \%){ }^{3}$ Both should be measured in all cases of suspected giant cell arteritis.

Scalp necrosis is a rare but known complication of giant cell arteritis and may be the presenting feature, before any visual loss. Lesions typically occur in the temporoparietal areas and resolve with systemic steroid therapy. Patients with scalp necrosis are usually older and are more likely to have an associated visual loss than those without scalp involvement, perhaps because scalp occlusive vasculitis or a delayed recognition of giant cell arteritis.

Scalp necrosis may be misdiagnosed as herpes zoster. ${ }^{1}$ However, herpes zoster is typically unilateral with numerous, discrete but characteristic crusted vesicular lesions, whereas the lesions of scalp necrosis associated with giant cell arteritis are usually large, more confluent and nonvesicular.

\section{Renatta Varma}

Anil D. Patel

Ophthalmology Department

University of Saskatchewan

Eye Care Centre

Saskatoon City Hospital

Saskatoon, Sask.

\section{References}

1. Currey J. Scalp necrosis in giant cell arteritis and review of the literature. Br7 Rheumatol 1997;36:814-6.

2. Ghanchi FD. Current concepts in giant cell (temporal) arteritis. Surv Ophthalmol 1997;42:99-123.

3. Hayreh SS, Podhajsky PA, Raman R, Zimmerman B. Giant cell arteritis: validity and reliability of various diagnostic criteria. Am 7 Ophthalmol 1997; 123:285-96. necrosis represents a more severe 